\title{
Cryptosporidium sp. em intestinos, bursa de Fabricius e traquéia de frangos (Gallus gallus)
}

\author{
Cryptosporidium sp. in intestines, bursa of Fabricius and poultry trachea (Gallus gallus)
}

\author{
Gislaine Jacobsen $^{1}$ Aléverson da Silva Barcelos ${ }^{2}$ Maristela Lovato Flôres ${ }^{3}$ \\ Stefanie Dickel Segabinazi ${ }^{4}$ Vera Regina Albuquerque Lagaggio ${ }^{5}$
}

\section{- NOTA -}

\section{RESUMO}

Parasitas do gênero Cryptosporidium infectam várias espécies de animais, e a enfermidade resultante é a criptosporidiose, importante zoonose de distribuição mundial. Em aves, a infecção tem sido reportada em várias espécies. Este trabalho objetivou identificar a presença do parasita em 208 amostras de bursa de Fabricius, 208 amostras de intestino e 208 de traquéia, coletadas de frangos (Gallus gallus) de diferentes idades, abatidos em três propriedades rurais do município de Santa Maria, RS. Foram feitas três impressões de cada amostra em lâminas para microscopia, coradas pelas técnicas de Ziehl Neelsen modificada com Dimetil Sulfóxido (DMSO), Ziehl Neelsen modificada por Henriksen e Pohlens (HP), Ziehl Neelsen (ZN) e Kinyoun (K), perfazendo 1872 impressões analisadas em microscopia óptica (1000 x). Neste total, nas diferentes colorações empregadas, oocistos do parasita Cryptosporidium sp. foram visualizados em 18 impressões de traquéia, 42 de bursa de Fabricius e 29 de intestino, resultando positivas, portanto, 89 impressões. Destas, 44 foram identificadas pela técnica de DMSO, 32 por HP, três por ZN e 10 por K. Pode-se concluir que os oocistos do parasita Cryptosporidium sp. foram visualizados com maior freqüencia nas impressões de bursa de Fabricius, e que o método de coloração, dentre os utilizados, que proporcionou a maior visualização dos oocistos foi o DMSO.

Palavras-chave: Cryptosporidium sp., frango, intestinos, bursa de Fabricius, traquéia.

\begin{abstract}
Parasites of the gennus Cryptosporidium infect several animal species.The disease resultant is the criptosporidiosis, an important zoonosis spreaded worldwide. In poultry, the infection has been reported in several species. This study goal was to identify the parasite presence in 208 bursa of Fabricius samples, 208 intestine and 208 of trachea, collected at chicks (Gallus gallus) of different ages, killed at three farms in Santa Maria city, Rio Grande do Sul State, Brazil. Three printings of each sample were done,on glass slides colored through the Ziehl Neelsen technique modified with Dimetil Sulfóxido (DMSO), Ziehl Neelsen modifed by Henriksen and Pohlens (HP), Ziehl Neelsen (ZN) and Kinyoun (K), completing 1872 printings analyzed in optical microscopy $(1000 \mathrm{x})$. At this final number, at the different colorations done, oocysts of the parasite Cryptosporidium sp. were viewed in 18 trachea printings, 42 of bursa de Fabricius and 29 of intestine, resulting positive, 89 printings. From these 89 , 44 were identified through the technique DMSO, 32 through HP, three through ZN and 10 through K. Cryptosporidium sp. parasites oocysts were found with more frequency from printings of bursa the Fabricius. The coloration method, of those utilized, was the one which had the better visualization of the oocysts was the DMSO.
\end{abstract}

Key words: Cryptosporidium sp., chicks, intestines, bursa of Fabricius, trachea.

\footnotetext{
'Programa de Pós-graduação em Medicina Veterinária (PPGMV), Universidade Federal de Santa Maria (UFSM), Santa Maria, RS, Brasil.

${ }^{2}$ Autônomo, Cruz Alta, RS, Brasil.

${ }^{3}$ Laboratório Central de Diagnóstico de Patologias Aviárias (LCDPA), Departamento de Medicina Veterinária Preventiva, Centro de Ciências Rurais, UfSM, Santa Maria, RS, Brasil. Prédio 44, sala 5151, 97105-900, Santa Maria, RS, Brasil. E-mail: patoaves@ccr.ufsm.br. Autor para correspondência.

${ }^{4}$ Departamento de Microbiologia e Parasitologia, Centro de Ciências da Saúde, UFSM, Santa Maria, RS, Brasil.

${ }^{5}$ Curso de Doutorado em Biologia Parasitária, Fundação Oswaldo Cruz, Rio de Janeiro, RJ, Brasil.
} 
O Cryptosporidium sp. é um parasita coccídio encontrado na região das microvilosidades do trato respiratório dos vertebrados. A taxonomia desse protozoário em relação à infecção das aves ainda é controversa, havendo definição de duas espécies, $\boldsymbol{C}$. meleagridis (SLAVIN, 1955) e C.baileyi (CURRENT et al., 1986). Também foi descrita recentemente a espécie C. galli parasitando aves exóticas e selvagens (RYAN et al., 2003). A infecção causada pelo protozoário é a criptosporidiose, a qual tem sido reportada em diversas espécies de aves, caracterizada por distúrbios respiratórios e intestinais (CHERMETTE \& BOUFASSA-OUZROUT,1988).

A criptosporidiose é uma importante enfermidade imunossupressora nas aves por determinar destruição do epitélio bursal, perda de microambiente e atrofia da bursa de Fabricius, também é considerada uma zoonose, pois bovinos, aves, cães, gatos e animais de laboratório constituem fonte de infecção para o homem (ITO, 1995). Na maioria dos casos de infecções naturais em frangos, o parasita foi encontrado principalmente na traquéia, bursa de Fabricius, cloaca, em menor grau no ceco e reto, e raramente no intestino (SRÉTER \& VARGA, 2000). Em pintos, perus e codornas, este parasita é primariamente patogênico, produzindo problemas respiratórios e intestinais (SAIF, 2003).

Após sua primeira descrição em aves por TYZZER (1929), até recentemente, a criptosporidiose foi considerada rara e oportunista. Entretanto a partir da década de 70 o Cryptosporidium sp. tem apresentado destaque crescente como agente etiológico de infecções envolvendo principalmente os tratos digestivo e respiratório de várias espécies. Contudo, foi somente após o seu reconhecimento como agente primário de doença clínica no homem e nos animais que o parasita passou a ter uma importância nunca antes experimentada (GOODWIN, 1989).

Este trabalho objetivou pesquisar a presença de oocistos do parasita Cryptosporidium sp. em 208 amostras de intestino, 208 bursas de Fabricius e 208 traquéias de frangos (Gallus gallus) de diferentes idades, abatidos para o consumo, coletadas em três propriedades rurais do município de Santa Maria-RS, bem como avaliar a eficiência das diferentes técnicas empregadas na coloração das impressões em lâminas para visualização do mesmo. Não foram observados sinais clínicos nas aves das quais as amostras foram coletadas, corroborando com SALTANOVA et al. (1991) em inoculação experimental por Cryptosporidium sp., em que relatam que as aves inoculadas eliminaram os oocistos durante um curto período de tempo e não desenvolveram sinais clínicos.

No Laboratório Central de Diagnóstico de Patologias Aviárias (LCDPA) da Universidade Federal de Santa Maria, foram feitas três impressões de cada amostra em lâmina para microscopia, coradas pelos métodos de Ziehl Neelsen modificado com Dimetil sulfóxido (DMSO), Ziehl Neelsen modificado por Henriksen e Pohlens, Ziehl Neelsen e Kinyon, conforme demonstra a tabela 1. Os oocistos e esporocistos são transparentes e de difícil visualização em esfregaços não corados e somente são identificados através de colorações específicas. Devido às limitações do exame microscópico de preparações a fresco, vários métodos de coloração favorecem o diagnóstico dos oocistos de coccídios (DE CARLI \& MOURA, 2000).

As impressões em lâminas foram examinadas ao microscópio ótico $(1.000 \mathrm{x})$ para observação de

Tabela 1 - Achados de oocistos de parasitas do gênero Cryptosporidium em impressões em lâminas para microscopia constituídas de amostras de traquéia, bursa de Fabricius e intestino de frangos (Gallus gallus), utilizando-se diferentes técnicas de coloração.

\begin{tabular}{|c|c|c|c|c|c|c|c|c|}
\hline \multirow{3}{*}{ Técnica } & \multicolumn{6}{|c|}{ Impressões } & & \\
\hline & \multicolumn{2}{|c|}{ Traquéia } & \multicolumn{2}{|c|}{ Bursa } & \multicolumn{2}{|c|}{ Intestino } & \multicolumn{2}{|c|}{ Total } \\
\hline & $\mathrm{n}$ & $\mathrm{p}$ & $\mathrm{n}$ & $\mathrm{p}$ & $\mathrm{n}$ & $\mathrm{p}$ & $\mathrm{N}$ & $\mathrm{P}$ \\
\hline Dimetil Sulfóxido & 208 & 09 & 208 & 20 & 208 & 15 & 624 & 44 \\
\hline Henriksen e Pohlens & 208 & 07 & 208 & 14 & 208 & 11 & 624 & 32 \\
\hline Ziehl Neelsen & 100 & 01 & 100 & 01 & 100 & 01 & 300 & 03 \\
\hline Kinyoun & 108 & 01 & 108 & 07 & 108 & 02 & 324 & 10 \\
\hline Total & 624 & 18 & 624 & 42 & 624 & 29 & 1872 & 89 \\
\hline
\end{tabular}

n= número de impressões em lâminas para microscopia, nos diferentes órgãos, nas diferentes técnicas empregadas.

$\mathrm{p}=$ número de impressões positivas, em lâminas para microscopia, nos diferentes órgãos, nas diferentes técnicas empregadas.

$\mathrm{N}=$ número total de impressões em lâminas para microscopia, nas diferentes técnicas empregadas.

$\mathrm{P}=$ número total de impressões positivas, em lâminas para microscopia, nas diferentes técnicas empregadas.

Ciência Rural, v.36, n.2, mar-abr, 2006. 
oocistos do parasita nas suas características usuais, sendo considerado resultado positivo apenas a presença dos mesmos, independentemente do número encontrado. No exame destas, foram observados oocistos arredondados com diâmetro de 3 a $5 \mu \mathrm{m}$ e coloração variando do rosa ao vermelho com áreas claras internamente. Oocistos de Cryptosporidium sp. são visualizados como estruturas de coloração rosa à púrpura intenso, com uma zona clara entre os esporocistos corados e a parede do oocisto (DE CARLI \& MOURA, 2000). Ainda, segundo SODRÉ \& FRANCO (2001), os oocistos são observados como estruturas esféricas ou ovaladas medindo aproximadamente $5 \mu \mathrm{m}$.

Ao todo, foram avaliadas 1.872 impressões em lâminas para microscopia, sendo, de cada um dos órgãos estudados, ou seja, traquéias, bursas de Fabricius e intestinos de frangos, 624 lâminas. Oocistos do parasita Cryptosporidium sp. foram visualizados em 18 impressões de traquéia, 42 de bursa de Fabricius e 29 de intestinos. Dessa forma resultaram positivas 89 impressões, representando $4,7 \%$ do total avaliado, independentemente da metodologia de coloração empregada (Tabela 1).

O aparelho respiratório serve de porta de entrada do parasita e, portanto, espera-se encontrá-lo em órgãos como a traquéia, pois este é o órgão primário de infecção (GOODWIN, 1989). Cryptosporidium sp. é um protozoário que causa doença respiratória em aves e, adicionalmente à traquéia, outros órgãos como o intestino e bursa de Fabricius podem sofrer infestação pelo parasita (TAKANO et al., 1992). A infestação ocorre com a ingestão de alimentos ou água contaminada com oocistos infectantes FERNÁNDEZ et al. (1990), podendo servir como fonte primária de distribuição de oocistos entre lotes de frangos (SRÉTER \&VARGA, 2000).

Aproximadamente $20 \%$ dos oocistos de Cryptosporidium sp. não formam uma parede de duas camadas resistente ao meio ambiente externo e sim uma única membrana envolvendo os quatro esporozoítos. Esses oocistos de parede delgada sofrem excistação ainda no hospedeiro, sendo responsáveis, juntamente com os merozoítos de primeira geração por autoinfecção que pode ser a origem do parasitismo intenso observado por vários dias na bursa de Fabricius (CURRENT et al., 1986).

A metodologia de coloração utilizada e que proporcionou uma maior repetição na visualização dos oocistos do parasita foi a de Ziehl Neelsen modificada com Dimetil Sulfóxido (DMSO), pois, através desta, oocistos foram visualizados em $49,5 \%$ do total de impresões positivas. Entretanto, a identificação definitiva só é possível com a utilização de anticorpos monoclonais (GOODWIN, 1989).
De acordo com os resultados obtidos e considerando as condições utilizadas nesta pesquisa verificou-se que, nas impressões de bursa de Fabricius, os oocistos do parasita Cryptosporidium sp. foram visualizados com maior freqüência, e que o método de coloração, dentre os utilizados, que proporcionou a maior visualização dos oocistos foi a DMSO. Considerando-se que o Gallus gallus constitui uma significativa fonte de divisas para o Brasil, e que o parasita é agente primário de doença clínica no homem, sua identificação em aves clinicamente saudáveis, como no presente trabalho, é de imensurável relevância.

\section{REFERÊNCIAS}

CHERMETTE, R.; BOUFASSA-OUZROUT, S. Cryptosporidiosis a cosmopolitan disease in animal and in man. 2.ed. Paris: OIE, 1988. 122p.

CURRENT, W.L. et al. The life cycle of Cryptosporidium baileyi. (Apicomplexa) Cryptosporididae infecting chickens. Journal of Protozoology, v.33, p.289-296, 1986.

DE CARLI, G.A.; MOURA, H. Parasitologia clínica: diagnóstico de laboratório dos coccídios e microsporídios intestinais. Porto Alegre: EDIPUCRS, 2000. 73p.

FERNÁNDEZ, A. et al. Cryptosporidiosis in chickens from southern Spain. Avian Diseases, v.34, p.224-227, 1990.

GOODWIN, M.A. Cryptosporidium in birds: a review. Avian Pathology, v.18, p.365-384, 1989.

ITO, N.M. Clipping de patologia aviária: doenças imunossupressoras das aves. São Paulo : Laboratório Pfizer do Brasil, 1995. 38p.

RYAN, U.M. et al. A redescription of Criptosporidium galli Pavlasek, 1999 (Apicomplexa: Cryptosporidiidae) from birds. Journal of Parasitology, v.89, p.809-813, 2003.

SAIF, Y.M. Disease of poultry. 11.ed. Ames: Iowa State, 2003. 1231p.

SALTANOVA, N.P. et al. The immune status of chicks experimentally infected with cryptosporidia. VeterinariyaMoskva, v.12, p.38-40, 1991.

SLAVIN, D. Cryptosporidium meleagridis. Journal of Comparative Pathology, v.65, p.262-266, 1955.

SODRÉ, F.C.; FRANCO, R.M.B. Novos aspectos sobre um tema bem conhecido: Cryptosporidium. Revista Brasileira de Análises Clínicas, v.33, p.97-104, 2001.

SRÉTER, T.; VARGA, I. Cryptosporidiosis in birds- a review. Veterinary Parasitology, v.87, p.261-279, 2000.

TAKANO, H. et al. Developmental process of cryptosporidium in the intestine and bursa de Fabricius of chickens. Journal of Veterinary Medicine Science, v.54, p.289-292, 1992.

TYZZER, E.E. Coccidiosis in gallinaceous birds. American Journal of Hygiene, v.10, p.269-383, 1929. 